\title{
Cinética e morfologia de espermatozoides de carpa comum criopreservados em água de coco em pó ACP-104
}

\author{
[Kinetics and morphology of common carp sperm cryopreserved in \\ powdered coconut water ACP-104] \\ F.R.A. Linhares, C.S.B. Salmito-Vanderley*, M.A.M. Carvalho, R.R.R. Pinheiro, \\ F.C.E. Oliveira, J.F. Nunes \\ Universidade Estadual do Ceará - UECE - Fortaleza, CE
}

\begin{abstract}
RESUMO
O objetivo deste estudo foi avaliar o efeito de diferentes taxas de diluição seminal sobre a motilidade, velocidade e presença de anormalidades espermáticas no sêmen descongelado de carpa comum (Cyprinus carpio). Utilizaram-se 20 machos sexualmente maduros, pertencentes ao Departamento Nacional de Obras Contra as Secas (DNOCS). A partir do sêmen coletado, foi formado um total de nove pools. O sêmen de cada pool foi avaliado quanto à motilidade, velocidade e morfologia espermática antes e depois da criopreservação. A criopreservação seminal foi realizada em meio ACP-104 + DMSO 10\% diluído em 1:1 ou 1:3 (sêmen:diluidor). As amostras foram envasadas em palhetas de $0,25 \mathrm{~mL}$, congeladas em vapor de nitrogênio líquido (caixa de poliestireno) e estocadas em nitrogênio líquido $\left(-196^{\circ} \mathrm{C}\right)$. Não houve diferença significativa $(\mathrm{P}>0,05)$ entre as taxas de diluições sobre os parâmetros cinéticos e morfológicos de sêmen descongelado de carpa comum. Entretanto, esses parâmetros apresentaram diferença significativa $(\mathrm{P}<0,05)$ em relação ao sêmen fresco (controle). Baixos valores de velocidade foram registrados no sêmen descongelado (VCL 42,6-46,5 $\mu \mathrm{m} / \mathrm{s}$; VSL 33,2-37,1 $\mu \mathrm{m} / \mathrm{s}$; VAP $38,9-43,2 \mu \mathrm{m} / \mathrm{s}$ ); contudo, observaram-se motilidades acima de 59\% (59,8-67,8\%) e baixos índices de anormalidades espermáticas (19,3-19,5\%), sugerindo que o ACP pode ser usado como um meio favorável à criopreservação do sêmen de carpa comum em qualquer uma das taxas de diluição testadas.
\end{abstract}

Palavras-chave: Cyprinus carpio, sêmen, criopreservação, motilidade espermática

\begin{abstract}
The aim of this study was to determine the effects of various dilution ratios on thaw sperm motility, velocity and presence of spermatic abnormalities in the common carp. 20 males with three years of age, raised at the Departamento Nacional de Obras Contra as Secas (DNOCS) were used. From the collected semen a total of nine pools were formed. The semen from each pool was evaluated for motility, velocity and spermatic morphology before and after cryopreservation. The sperm cryopreservation was performed in medium ACP-104 + DMSO 10\% diluted in 1:1 or 1:3 (sperm:extender). The samples were loaded into 0.25mL straws, frozen in liquid nitrogen vapor (Styrofoam) and stored in liquid nitrogen ($\left.196{ }^{\circ} \mathrm{C}\right)$. There was no significant difference $(P>0.05)$ between the ratios of dilution on the kinetic and morphological parameters of thawed semen from common carp. However, these parameters were significantly different $(P<0.05)$ compared to fresh semen (control). Low velocity values were recorded in

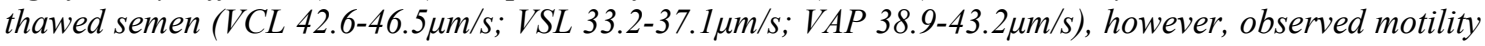
above 59\% (59.8-67.8\%), and low levels of spermatic abnormalities (19.3-19.5\%) suggested that the ACP-104 can be used as a suitable medium for cryopreservation of common carp semen in any of the tested dilution ratios.
\end{abstract}

Keywords: Cyprinus carpio, sperm, cryopreservation, sperm motility

Recebido em 7 de outubro de 2014

Aceito em 18 de junho de 2015

*Autor para correspondência (corresponding author)

E-mail: sandra.salmito@uece.br 


\section{INTRODUÇÃO}

A criopreservação seminal é uma biotecnia conhecida por resultar em consideráveis danos à estrutura celular. Sendo assim, um dos principais objetivos das pesquisas tem sido a elaboração de um protocolo ideal para minimizar os danos provocados às células espermáticas durante $\mathrm{o}$ congelamento e descongelamento do sêmen.

Desse modo, a adição de diluidores ao sêmen se tornou uma etapa imprescindível no processo, uma vez que o congelamento de sêmen puro é praticamente inviável por causar danos a grande parte dos espermatozoides (Salmito-Vanderley et al., 2012). Em geral, essas soluções devem ser compostas por uma substância para diluir e nutrir as células, e crioprotetores, para protegê-las contra a formação de cristais de gelo, flutuações do $\mathrm{pH}$ e choque osmótico (Muchlisin, 2004).

Outro fator a ser levado em consideração é a verificação da melhor proporção entre as quantidades de diluidor e sêmen (taxa de diluição). Öğretmen et al. (2015) afirmam que a diluição reduz os componentes do plasma seminal, tornando as células mais sensíveis ao estresse oxidativo. Por outro lado, a diluição é fundamental para maximizar o volume do material fecundante (Yang e Tiersch, 2009). Portanto, é importante avaliar quanto de diluente pode ser acrescentado ao sêmen sem lhe causar prejuízos após o descongelamento.

Vale ressaltar ainda que a concentração do crioprotetor também deve ser considerada durante a elaboração de um diluidor seminal. Sabe-se que o DMSO na concentração a $10 \%$ tem sido o crioprotetor mais testado e com melhores resultados de motilidade quando associado a diferentes diluidores (Irawan et al., 2010; Yildiz et al., 2015). Contudo não se conhecem os resultados da interação da água de coco com o DMSO em diferentes taxas de diluição sobre a cinética e morfologia de espermatozoides de carpa criopreservados em ACP-104.

A água de coco em pó (ACP-104) adaptada para a criopreservação de sêmen de peixes é um diluente seminal que vem ganhando destaque em espécies nativas brasileiras (Viveiros et al., 2008; Leite et al., 2011). Assim, o seu uso como diluidor seminal de carpa poderá contribuir para a sua disseminação no mundo, uma vez que a espécie é cultivada em muitos países.

Desse modo, este trabalho teve como objetivo principal testar duas taxas de diluição (1:1 e 1:3), analisando seus efeitos através da cinética espermática (motilidade e velocidade) e da presença de anormalidades espermáticas.

\section{MATERIAL E MÉTODOS}

A pesquisa foi aprovada pelo Comitê de Ética para o Uso de Animais da UECE (protocolo $\mathrm{n}^{\circ}$ 11516665-3/63). Para tanto, vinte machos de carpa comum, com idade média de três anos, comprimento médio de $60 \mathrm{~cm}$ e peso médio de $3,0 \mathrm{~kg}$, foram selecionados e mantidos em viveiros de terra com $350 \mathrm{~m}^{2}$ (temperatura média de $27,5^{\circ} \mathrm{C}$ ), no Centro de Pesquisas em Aquicultura (CPAq), pertencente ao Departamento Nacional de Obras Contra as Secas (DNOCS) (Pentecoste - Ceará), local onde se deram as coletas de sêmen e as primeiras avaliações seminais.

Os procedimentos iniciais de aferição do aspecto, $\mathrm{pH}$, volume e motilidade subjetiva foram realizados em Pentecoste, enquanto as análises subsequentes de osmolaridade, concentração, morfologia, motilidade objetiva e velocidades espermáticas foram realizadas no Laboratório de Biotecnologia da Reprodução de Peixes do Núcleo Integrado de Biotecnologia (LBRP-NIB) da UECE (Fortaleza, Ceará, Brasil) após duas horas de transporte.

Os animais que liberavam sêmen a partir de uma leve massagem abdominal foram selecionados, pesados e medidos para receber aplicação única de extrato hipofisário de carpa (Danúbio Aquicultura Ltda., SC, Brasil) na proporção de $1 \mathrm{mg} / \mathrm{Kg}$ de peso vivo, por via intracelomática, na base da nadadeira peitoral. Doze horas após a indução hormonal da espermiação, os animais foram previamente anestesiados por meio de imersão em solução contendo Eugenol (Eugenol, Cequímica Ltda.) diluído em álcool absoluto e água $(1: 10: 10000)$ até a perda de equilíbrio. Em seguida, cada animal foi mantido em decúbito lateral e seu orifício urogenital foi enxuto com papel toalha para evitar a contaminação do sêmen. Este foi coletado em tubos de polietileno, realizando-se uma pressão abdominal no sentido anteroposterior. 
Durante as análises seminais, as amostras mantiveram-se conservadas em caixa térmica $\left(\sim 5^{\circ} \mathrm{C}\right)$. $\mathrm{O}$ volume seminal foi aferido por meio de tubos graduados. $O$ aspecto seminal foi observado quanto à coloração e viscosidade, considerando-se satisfatórios branco-cremoso ou branco-leitoso (Vieira et al., 2011). O pH foi obtido através da deposição de $2 \mu \mathrm{L}$ de sêmen sobre as fitas de $\mathrm{pH}$ (MERCK-Alemanha). A análise microscópica subjetiva da motilidade foi estimada de cada animal $(n=20)$, utilizando um microscópio ótico (Nikon Eclipse E200 ampliação de 400x) após a mistura de uma alíquota de $2 \mu \mathrm{L}$ de sêmen e $100 \mu \mathrm{L}$ de água do tanque de manejo, considerando-se uma escala de $0 \%$ (nenhum espermatozoide móvel) a 100\% (todos os espermatozoides móveis). Foram utilizadas no experimento somente as amostras com o valor superior a $80 \%$ de motilidade, enquanto aquelas contaminadas com água, fezes, urina ou sangue foram descartadas.

As amostras de sêmen dos 20 animais foram utilizadas para a formação de nove pools. Cada pool foi constituído pela combinação das amostras de três machos de forma aleatória, em que sete animais contribuíram mais de uma vez para a formação dos pools. Os pools foram divididos em duas alíquotas e diluídos na proporção 1:1 ou 1:3 (sêmen:diluidor) em diluidor ACP-104 (ACP Biotecnologia, Fortaleza, Ceará, Brasil) acrescido de DMSO $10 \%$. A preparação da solução crioprotetora foi realizada da seguinte maneira: $50 \mathrm{~mL}$ de água destilada foram misturados a $3,46 \mathrm{~g}$ do pó ACP$104\left(\mathrm{pH} 8,0 ; 300 \mathrm{mOsmol} . \mathrm{kg}^{-1}\right)$, sendo retirados $10 \mathrm{~mL}$ da solução preparada e acrescentados $10 \mathrm{~mL}$ de DMSO (315mOsm. $\mathrm{Kg}^{1} \quad \mathrm{H}_{2} \mathrm{O}$, Cequímica Ltda., Brasil). Durante as diluições, o sêmen e os crioprotetores permaneceram refrigerados $\left(\sim 5^{\circ} \mathrm{C}\right)$.

Simultaneamente, três litros $(5 \mathrm{~cm}$ de profundidade) de nitrogênio líquido foram despejados numa caixa térmica de poliestireno com dimensões de $40 \mathrm{~cm}$ de comprimento, $24 \mathrm{~cm}$ de largura e $28 \mathrm{~cm}$ de altura. Após estabilização (5-10min.) da temperatura no interior dessa caixa $\left(-100^{\circ} \mathrm{C}\right)$, os dois tratamentos foram envasados em palhetas francesas de $0,25 \mathrm{~mL}$, distribuídas em raques e colocadas horizontalmente sobre um suporte feito de malha de metal a três centímetros da superfície do nitrogênio líquido.
A caixa de poliestireno foi fechada com no máximo quatro raques no seu interior para evitar variações bruscas de temperatura. Assim, as amostras permaneceram no interior da caixa por 10min, mantendo-se a uma temperatura média interna de $-118^{\circ} \mathrm{C}$ (termômetro de sonda Headerterm 200, tipo Pt-100, Header Instrumentos Ltda.). Em seguida, as amostras foram transferidas para um botijão de nitrogênio líquido $\left(-196^{\circ} \mathrm{C}\right)$ e transportadas do DNOCS, Pentecoste, até o LBRP-NIB, Fortaleza, onde foram estocados por uma semana. Um total de 108 palhetas foram criopreservadas, resultantes da combinação de 6 palhetas x 2 tratamentos x 9 pools.

A osmolaridade $(\mathrm{mOsm} / \mathrm{kg})$ dos nove pools foi mensurada por meio de osmômetro digital de refrigeração Peltier (Roebling, Alemanha). Para a determinação da concentração espermática, $1 \mu \mathrm{L}$ de sêmen foi diluído em $4 \mathrm{~mL}$ de solução citrato-formol 4\%. Dessa mistura, uma alíquota de $20 \mu \mathrm{L}$ foi depositada em câmara de Neubauer para análise em microscópio ótico (400x). Após 15 minutos de sedimentação da amostra, as contagens foram realizadas em cinco quadrantes nos dois retículos, sendo descartadas leituras que diferiram de $10 \%$ entre os retículos. Foram realizadas quatro repetições por amostra. As médias do número de células contadas nos dois retículos foram multiplicadas pelo fator $200 \mathrm{x}$ $10^{6}$, segundo Akçay et al. (2004).

Para a análise morfológica dos espermatozoides, foram realizados esfregaços com azul de bromofenol (1g azul de bromofenol; $4 \mathrm{~g}$ citrato de sódio; $100 \mathrm{~mL}$ água destilada; $\mathrm{pH} 8,0$ ) a partir da diluição do sêmen fresco (1:500) e descongelado (1:250) em solução citrato-formol a 4\%. Em um tubo de polietileno, misturou-se uma alíquota de $6 \mu \mathrm{L}$ da solução de fixação com sêmen fresco ou descongelado com $6 \mu \mathrm{L}$ de azul de bromofenol. Dessa mistura, foram depositadas alíquotas de $4 \mu \mathrm{L}$ em duas lâminas para a realização das leituras em microscópio ótico (Nikon 102 Eclipse E200 - 400x). Assim, para cada tratamento, foram confeccionadas duas lâminas, em que foram observados 100 espermatozoides por lâmina.

Os espermatozoides foram classificados como ou normais ou presença de anormalidades primárias (macrocefalia, microcefalia, cabeça degenerada, peça intermediária degenerada, cauda quebrada, 
cauda pequena e cauda fortemente enrolada) ou anormalidades secundárias (cabeça normal sem cauda, cauda levemente dobrada, gota proximal, gota distal), conforme metodologia utilizada por Miliorini et al. (2011).

A análise objetiva dos parâmetros cinéticos do sêmen fresco (9 pools), considerado grupo controle, foi realizada por meio do sistema de Análise Seminal Auxiliada por Computador (CASA), avaliando-se: espermatozoides móveis totais (\%), considerando todos os espermatozoides com velocidade superior a $10 \mu \mathrm{m} / \mathrm{s}$, velocidade curvilinear (VCL $-\mu \mathrm{m} / \mathrm{s}$ ), velocidade em linha reta (VSL - $\mu \mathrm{m} / \mathrm{s}$ ) e velocidade média do percurso (VAP - $\mu \mathrm{m} / \mathrm{s}$ ). Para tanto, a trajetória de cada espermatozoide (pelo menos 500 espermatozoides/palheta) foi calculada ao longo das 25 imagens/s geradas.

Para essa análise, uma alíquota de $2 \mu \mathrm{L}$ de sêmen fresco foi misturada com $100 \mu \mathrm{L}$ de solução ativadora $\mathrm{NaCl}$ (100mOsm) em câmara de Makler $^{\circledR}$ e analisada por meio de um microscópio ótico com contraste de fase (Nikon ${ }^{\circledR}$ H550S, ECLIPSE 50i, Japan), numa ampliação de 400x, com o filtro verde e contraste de fase em posição pH1. O microscópio conectado a uma câmera de vídeo (Basler Vision Technologies ${ }^{\circledR}$ A312FC, Ahrensburg, Alemanha) gerava 25 imagens/s. A gravação do vídeo foi iniciada de três a quatro segundos após a ativação. $\mathrm{O}$ vídeo foi analisado conforme a configuração padrão para peixes do software Sperm Class Analyzer ${ }^{\circledR}\left(\mathrm{SCA}^{\circledR}\right.$, Microptics-Barcelona - Espanha, versão 3.2).

Uma semana depois do processo de criopreservação, o sêmen foi descongelado em banho-maria a $25^{\circ} \mathrm{C}$ por 30 segundos e foram analisados os parâmetros cinéticos seguindo a mesma metodologia utilizada para o sêmen fresco.

Para a análise estatística, inicialmente os dados foram submetidos aos Testes de KolmogorovSmirnov e de Levene para confirmação da distribuição normal e homogeneidade de variância entre tratamentos, respectivamente. O parâmetro cauda enrolada só apresentou normalidade após transformação logarítmica na base 10. Confirmado o atendimento das exigências para realização da Análise de Variância (ANOVA), esta foi executada por meio do software SPSS versão 14.0 e o teste de Games-Howell foi empregado para comparação das médias. Os resultados foram expressos como média \pm desvio padrão, e as diferenças foram consideradas significativas quando $\mathrm{P}<0,05$.

\section{RESULTADOS E DISCUSSÃO}

Das amostras de sêmen dos 20 animais selecionados, 15 apresentaram coloração branco leitoso, enquanto cinco apresentaram coloração branco cremoso. A taxa média de motilidade subjetiva encontrada para o sêmen fresco foi de $97 \%$. A concentração espermática média encontrada foi de $20,68 \pm 2,85 \times 10^{9}$ espermatozoides $/ \mathrm{mL}$ e um volume médio de $28,45 \mathrm{~mL}$. A osmolaridade média foi igual a $277,6 \pm 21,2 \mathrm{mOsm} / \mathrm{Kg}$, e o $\mathrm{pH}$ igual a 8,52 .

No que diz respeito aos parâmetros cinéticos, foi observada uma redução significativa $(\mathrm{P}<0,05)$ na motilidade total do sêmen descongelado nas duas taxas de diluição testadas $1: 1(59,8 \pm 12,5 \%)$ e $1: 3$ $(67,8 \pm 10,1 \%)$, respectivamente, em comparação com o sêmen fresco $(97,1 \pm 2,0 \%)$ (Tab. 1). No entanto, não houve diferença significativa $(\mathrm{P}>0,05)$ entre as taxas de diluições 1:1 $(59,8 \pm 12,5 \%) \quad$ e $1: 3 \quad(67,8 \pm 10,1 \%)$ para motilidade total (Tab. 1). Assim, estes resultados confirmam o que a maioria dos estudos sobre criopreservação de sêmen tem observado em relação à perda da motilidade (Li et al., 2006). Essa redução da motilidade após a criopreservação pode estar correlacionada com danos morfológicos e estruturais dos espermatozoides, tais como: fragmentação de DNA, ruptura da membrana citoplasmática, intumescimento de cabeça, encurtamento do flagelo, entre outros, durante o processo de congelamento e descongelamento (Galo et al., 2011). 
Cinética e morfologia...

Tabela 1. Efeito de diferentes taxas de diluição sobre os parâmetros cinéticos de espermatozoides frescos (grupo controle) e descongelados de Cyprinus carpio criopreservados em ACP-104

\begin{tabular}{cccccc} 
& Diluições & \multicolumn{4}{c}{ Parâmetros espermáticos } \\
\cline { 3 - 6 } Diluente & (sêmen:diluente) & $\begin{array}{c}\text { Motilidade Total } \\
(\%)\end{array}$ & $\begin{array}{c}\text { VCL } \\
(\mu \mathrm{m} / \mathrm{s})\end{array}$ & $\begin{array}{c}\text { VSL } \\
(\mu \mathrm{m} / \mathrm{s})\end{array}$ & $\begin{array}{c}\text { VAP } \\
(\mu \mathrm{m} / \mathrm{s})\end{array}$ \\
\hline ACP-104+ & $1: 1$ & $59,8 \pm 12,5 \mathrm{~b}$ & $42,6 \pm 10,7 \mathrm{~b}$ & $33,2 \pm 9,5 \mathrm{~b}$ & $38,9 \pm 10,6 \mathrm{~b}$ \\
DMSO & $1: 3$ & $67,8 \pm 10,1 \mathrm{~b}$ & $46,5 \pm 7,3 \mathrm{~b}$ & $37,1 \pm 6,8 \mathrm{~b}$ & $43,2 \pm 7,6 \mathrm{~b}$ \\
Controle* & - & $97,1 \pm 2,0 \mathrm{a}$ & $68,9 \pm 20,7 \mathrm{a}$ & $29,7 \pm 9,1 \mathrm{a}$ & $45,8 \pm 13,2 \mathrm{a}$ \\
\hline
\end{tabular}

Letras minúsculas distintas, na mesma coluna, indicam diferença significativa entre os tratamentos $(\mathrm{P}<0,05)$. ACP104: água de coco em pó para sêmen de teleósteos de água doce de fertilização externa; DMSO: Dimetil sulfóxido; VCL: velocidade curvilinear; VSL: velocidade retilinear; VAP: velocidade média do percurso. ${ }^{*} \mathrm{O}$ controle corresponde ao sêmen fresco.

No entanto, não houve diferença significativa $(\mathrm{P}>0,05)$ entre as taxas de diluições $1: 1$ $(59,8 \pm 12,5 \%) \quad$ e $\quad 1: 3 \quad(67,8 \pm 10,1 \%) \quad$ para motilidade total (Tab. 1). A literatura relata que as taxas de diluições empregadas podem influenciar a qualidade do sêmen após o descongelamento (Muchlisin et al., 2010). Isso provavelmente está relacionado com a densidade espermática durante as diluições, ou seja, baixas taxas de diluições proporcionam maiores quantidades de espermatozoides, favorecendo maiores chances de sobrevivência espermática durante a criopreservação. Entretanto, a proximidade entre as baixas taxas de diluição empregadas não possibilitaram uma diferença significativa entre as densidades espermáticas, podendo ter sido a causa da pequena diferença entre ambas no que diz respeito à avaliação de motilidade espermática total.

Outro fator que deve ser levado em consideração durante o aumento da taxa de diluição é a perda da capacidade protetora das proteínas do plasma seminal sobre os espermatozoides (Billard, 1988). Provavelmente essa capacidade protetora não foi perdida devido às baixas taxas de diluição utilizadas neste trabalho.

Muitos estudos sobre a criopreservação do sêmen de carpa apontam que o sucesso é alcançado com taxas de diluição de 1:1 (Irawan et al., 2010), 1:9 (Sultana et al., 2010) e 1:5 (Linhart et al., 2000), obtendo motilidades superiores a $60 \%$ utilizando DMSO a $10 \%$ como crioprotetor. As diferentes taxas de motilidade observadas nesses estudos podem estar relacionadas ao tipo de protocolo adotado, uma vez que foram utilizados três tipos de diluentes nos três protocolos.

Em comparação com o sêmen fresco (VCL: $68,9 \pm 20,7 \mu \mathrm{m} / \mathrm{s}$, VSL: $29,7 \pm 9,1 \mu \mathrm{m} / \mathrm{s}$ e VAP:
$45,8 \pm 13,2 \mu \mathrm{m} / \mathrm{s})$, as velocidades de natação foram significativamente reduzidas no sêmen congelado e descongelado diluído em 1:1 (VCL: $42,6 \pm 10,7 \mu \mathrm{m} / \mathrm{s}$, VSL: $33,2 \pm 9,5 \mu \mathrm{m} / \mathrm{s}$ e VAP: $38,9 \pm 10,6 \mu \mathrm{m} / \mathrm{s}$ ) e $1: 3$ (VCL: $46,5 \pm 7,3 \mu \mathrm{m} / \mathrm{s}$, VSL: $37,1 \pm 6,8 \mu \mathrm{m} / \mathrm{s}$ e VAP: $43,2 \pm 7,6 \mu \mathrm{m} / \mathrm{s}$ ). Contudo, para o sêmen descongelado, as velocidades espermáticas não diferiram $(\mathrm{P}>0,05)$ entre as taxas de diluição 1:1 e 1:3 (Tab. 1).

Este é um estudo original sobre a utilização do ACP como diluente seminal de carpa comum. Os resultados aqui encontrados com o sêmen congelado em ACP foram superiores aos de outros trabalhos utilizando o mesmo diluidor em outras espécies (Carvalho et al., 2010), porém inferiores ao do estudo realizado por Viveiros et al. (2010), no qual obtiveram valores médios de velocidade espermática de VCL $53,7 \pm 25,1 \mu \mathrm{m} / \mathrm{s}$, VSL $23,6 \pm 8 \mu \mathrm{m} / \mathrm{s}$ e VAP $36,8 \pm 14,8 \mu \mathrm{m} / \mathrm{s}$. Além disso, o ACP equiparou-se ao diluente glicose utilizado como padrão na criopreservação seminal de curimba (Prochilodus lineatus) (VCL $49,1 \pm 19,3 \mu \mathrm{m} / \mathrm{s}$, VSL $24,3 \pm 7,4 \mu \mathrm{m} / \mathrm{s}$ e VAP $36,0 \pm 13,1 \mu \mathrm{m} / \mathrm{s}$ ), demonstrando que o mesmo é eficiente para a conservação de gametas de algumas espécies de peixes (Viveiros et al., 2010).

Isso porque a eficiência crioprotetora aumenta quando os constituintes criopreservantes apresentam uma combinação de ação interna e externa à célula espermática (Godinho et al., 2003). Em geral, os açúcares acrescidos aos criodiluentes atuam no recobrimento da superfície celular e estabilização da membrana, ajudando, portanto, a minimizar e reparar os possíveis danos celulares causados pelo processo de congelamento (Urbányi et al., 2006). 
$\mathrm{Na}$ presente pesquisa, independentemente das taxas de diluição testadas, o ACP, um composto também formado por açúcares, obteve taxas de motilidade semelhantes aos diluentes frutose, glicose, sacarose, $\mathrm{KCl}$ e Kurokura para a conservação de sêmen de diferentes espécies de ciprinídeos (Urbányi et al., 2006). Assim, com relação à motilidade total, os valores obtidos na presente pesquisa com sêmen descongelado de carpa utilizando ACP-104 podem fornecer resultados satisfatórios para taxa de fertilização, visto que valores inferiores de motilidade total relatados por Billard et al. (2004) obtiveram bons resultados de fertilização.

Embora o DMSO tenha sido eficaz neste estudo por apresentar elevada taxa de motilidade, algumas pesquisas revelaram que ele pode provocar um enrolamento em forma de laço na ponta do flagelo, influenciando negativamente os resultados de velocidade ( $\mathrm{Li}$ et al., 2010). Entretanto, Linhart et al. (2000) relataram que é a motilidade espermática, e não a velocidade, que limita a capacidade fertilizante dos espermatozoides. Dessa forma, sugere-se a realização de novas pesquisas para se avaliar outros crioprotetores e suas interações com o ACP.

O número de anormalidades espermáticas aumentou consideravelmente no sêmen descongelado, independentemente das taxas de diluição empregadas, e foi significativamente superior $(\mathrm{P}<0,05)$ às encontradas no sêmen fresco. Entretanto, entre as taxas de diluição 1:1 e 1:3 não houve diferença significativa (Tab. 2).

Tabela 2. Efeito de diferentes taxas de diluições sobre os parâmetros morfológicos de sêmen descongelado de Cyprinus carpio

\begin{tabular}{|c|c|c|c|c|c|}
\hline \multirow[b]{2}{*}{ Diluente } & \multirow{2}{*}{$\begin{array}{c}\text { Diluições } \\
\text { (sêmen:diluente) }\end{array}$} & \multicolumn{4}{|c|}{ Parâmetros morfológicos (\%) } \\
\hline & & Normal & Macrocefalia & $\begin{array}{c}\text { Cauda } \\
\text { fortemente } \\
\text { enrolada }\end{array}$ & $\begin{array}{c}\text { Cauda } \\
\text { quebrada }\end{array}$ \\
\hline ACP-104+ & $1: 1$ & $80,4 \pm 4,7^{b}$ & $4,5 \pm 2,9^{b}$ & $7,6 \pm 5,5^{b}$ & $7,4 \pm 3,8^{b}$ \\
\hline DMSO $10 \%$ & $1: 3$ & $81,4 \pm 2,8^{b}$ & $4,6 \pm 4,2^{b}$ & $6,2 \pm 6,3^{b}$ & $8,5 \pm 4,1^{b}$ \\
\hline Controle* & Sem diluição & $95,1 \pm 1,8^{\mathrm{a}}$ & $0,03 \pm 0,1^{\mathrm{a}}$ & $2,2 \pm 1,5^{\mathrm{a}}$ & $2,5 \pm 1,6^{\mathrm{a}}$ \\
\hline
\end{tabular}

Letras minúsculas distintas, na mesma coluna, indicam diferença significativa entre os tratamentos $(\mathrm{P}<0,05)$. ACP104: água de coco em pó para sêmen de teleósteos de água doce de fertilização externa; DMSO: Dimetil sulfóxido *O controle corresponde ao sêmen fresco.

Observou-se um total de $4,7 \%$ de anormalidades espermáticas com sêmen fresco e uma média de $19,4 \%$ de anormalidades espermáticas com sêmen descongelado. Esse aumento de $14,7 \%$ de alterações morfológicas espermáticas podem ter sido ocasionado pelas crioinjúrias durante o processo de congelamento e descongelamento do sêmen, ocasionando danos às estruturas celulares (Li et al., 2006). Além disso, baixas taxas de diluição empregadas na pesquisa podem ter causado compressão e danos aos espermatozoides (Lahnsteiner, 2000).

Apesar disso, a ocorrência de 19,4\% de patologias espermáticas encontradas no sêmen descongelado poderá representar uma parcela insignificante de espermatozoides anormais quando se considera uma espécie com elevada concentração espermática, como é o caso da carpa comum, que pode variar desde $9,63 \times 10^{9}$ a $2,74 \times 10^{10}$ espermatozoides/mL (Christ et al., 1996). Do exposto, testes de fertilização deverão ser realizados para se confirmar essa hipótese.

As anormalidades primárias foram as únicas observadas no sêmen fresco e descongelado, encontrando-se apenas macrocefalia, cauda fortemente enrolada e cauda quebrada (Fig. 1). 


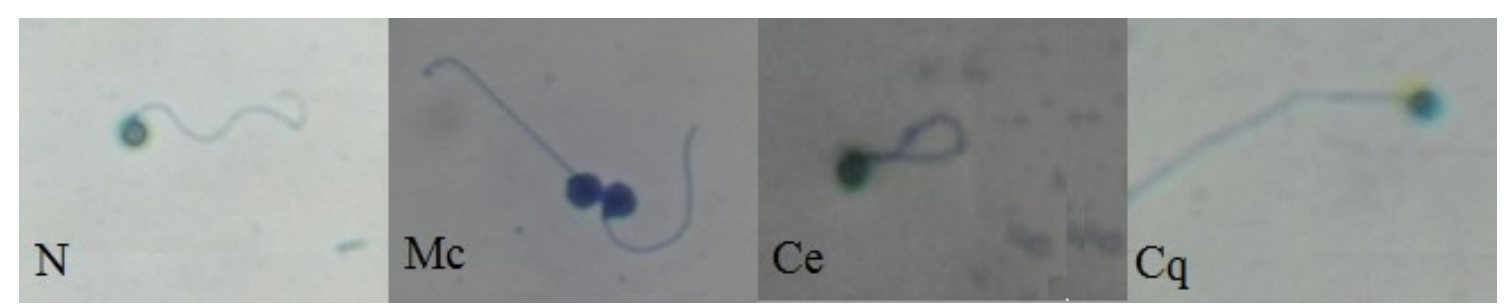

Figura 1. Morfologia espermática observada no sêmen fresco e descongelado de Cyprinus carpio depois de submetido a diferentes taxas de diluições. $\mathrm{N}$ - espermatozoide normal; Mc - espermatozoides com macrocefalia; $\mathrm{Ce}$ - espermatozoide com cauda fortemente enrolada; $\mathrm{Cq}$ - espermatozoide com cauda quebrada. Azul de bromofenol. Aumento x400.

\section{CONCLUSÃO}

Conclui-se que as taxas de diluição $1: 1$ e $1: 3$ podem ser empregadas para diluir o sêmen de carpa comum com ACP-104. Essa tecnologia poderá ser utilizada tanto em estudos de melhoramento genético quanto na formação de bancos de germoplasma através de programas de fertilização assistida.

\section{AGRADECIMENTOS}

Ao Departamento Nacional de Obras Contra as Secas (DNOCS) por disponibilizar laboratórios e estruturas de pesquisa, ao Conselho Nacional de Desenvolvimento Científico e Tecnológico (CNPq) pelo apoio financeiro, e à empresa $\mathrm{ACP}$ Biotecnologia pela doação da água de coco em pó (ACP-104).

\section{REFERÊNCIAS}

AKÇAY, E.; BOZKURT, Y.; SEÇER, S. et al. Cryopreservation of Mirror Carp Semen. Turk. J. Vet. Anim. Sci., v.28, p.837-843, 2004.

BILLARD, R. Artificial insemination and gamete management in fish. Mar. Behav. Physiol., v.14, p.3-21, 1988.

BILLARD, R.; COSSON, J.; NOVEIRI, S. B.; POURKAZEMI, M. Cryopreservation and shortterm storage of sturgeon sperm, a review. Aquaculture, v.236, p.1-9, 2004.

CARVALHO, M.A.M.; LINHARES, F.R.A.; NUNES, J.F. et al. Água de coco como diluente para o sêmen de peixes de água doce de fertilização externa. Rev. Bras. Hig. San. Anim., v.8, p.203-222, 2014.
CHRIST, S.A.; TOTH, G.P.; MCCARTHY, H.W. et al. Monthly variation in sperm motility in common carp assessed using computerassisted sperm analysis (CASA). J. Fish Biol., v.48, p.1210-1222, 1996.

GALO, J.M.; STREIT-JR., D.P.; SIROL, R.N. et al. Spermatic abnormalities of piracanjuba Brycon orbignyanus (Valenciennes, 1849) after cryopreservation. Braz. J. Biol., v.71, p.693-699, 2011.

GODINHO, H.P.; AMORIM, V.M.C.; PEIXOTO, M.T.D. Criopreservação do sêmen de tilápia-nilótica Oreochromis niloticus var. Chitralada: crioprotetores, soluções ativadoras e refrigerador criogênico. Rev. Bras. Zootec., v.32, p.1537-1543, 2003.

IRAWAN， H.; VUTHIPHANDCHAI， V.; NIMRAT, S. The effect of extenders, cryoprotectants and cryopreservation methods on common carp (Cyprinus carpio) sperm. Anim. Reprod Sci., v.122, p. 236-243, 2010.

LAHNSTEINER, F. Semen cryopreservation in the Salmonidae and in the northern pike. Aquacult. Res., v.31, p.245-258, 2000.

LEITE, L.V.; OLIVEIRA, F.C.E.; NUNES, L.T. et al. Criopreservação de sêmen de tambaqui com ACP adicionado de gema de ovo. Rev. Bras.Eng. Pesca, v.6, p. 23-29, 2011.

LI, J.; LIU, Q.; ZHANG, S. Evaluation of the damage in fish spermatozoa cryopreservation. Chinese J. Oceanol. Limnol., v.24, p.370-377, 2006.

LINHART, O.; RODINA, M.; COSSON, J. Cryopreservation of sperm in common carp Cyprinus carpio: Sperm motility and hatching success of embryos. Cryobiology, v.41, p. 241250,2000 . 
MILIORINI, A.B.; MURGAS, L.D.S.; ROSA, P.V. et al. A morphological classification proposal for curimba (Prochilodus lineatus) sperm damages after cryopreservation. Aquacult. Res., v.42, p.177-187, 2011.

MUCHLISIN, Z.A. Current status of extenders and cryoprotectants on fish spermatozoa cryopreservation. Biodiversitas, v.6, p.66-69, 2004.

MUCHLISIN, Z.A.; NADIYA, N.; NADIAH, W.N. et al. Preliminary study on the natural extenders for artificial breeding of African catfish Clarias gariepinus (Burchell, 1822). AACL Bioflux, v.3, p.119-124, 2010.

ÖĞRETMEN, F.; İNANAN, B.E.; KUTLUYER, F.; KAYIM, M. Effect of semen extender supplementation with cysteine on postthaw sperm quality, DNA damage, and fertilizing ability in the common carp (Cyprinus carpio). Theriogenology, v.83, p.1548-1552, 2015.

SALMITO-VANDERLEY, C.S.B.; VIEIRA, M.J.A. F; LEITE, L.V. et al. Meios de congelação para conservação de sêmen de peixes da família characidae. Ciênc. Anim., v.22, p.255268,2012 .

SULTANA, M.; NAHIDUZZAMAN, M.; HASSAN, M.M. et al. Fertility of cryopreserved common carp (Cyprinus carpio) spermatozoa. Rajshahi Univ. Zool. Soc., v.28, p.51-55, 2010.
URBÁNYI, B.; SZABÓ, T.; MISKOLCZI, E. et al. Successful fertilization and hatching of four European cyprinid species using cryopreserved sperm. J. Appl. Ichthyol., v.22, p.201-204, 2006.

VIEIRA, M.J.A.F.; CARVALHO, M.A.M.; SALMITO-VANDERLEY, C.S.B. et al. Características do sêmen de tambaqui (Colossoma macropomum) em latitude equatorial. Arch. Zootec., v.60, p. 1270, 2011.

VIVEIROS, A.T.M.; MARIA, A.M.; ORFÃO, L.H. et al. Powder coconut water (ACP) as extender for semen cryopreservation of Brazilian migratory fish species. Cybium, v.32, p.137, 2008.

VIVEIROS, A.T.M.; NASCIMENTO, A.F.; ORFÃO, L.H. et al. Motility and fertility of the subtropical freshwater fish streaked prochilod (Prochilodus lineatus) sperm cryopreserved in powdered coconut water. Theriogenology, v.74, p.551-556, 2010.

YANG, H.; TIERSCH, T.R. Current status of sperm cryopreservation in biomedical research fish models: zebrafish, medaka, and Xiphophorus. Comp. Biochem. Physiol. C, v.149, p.224-232, 2009.

YILDIZ, C.; YAVAS, I.; BOZKURT, Y.; AKSOY, M. Effect of cholesterol-loaded cyclodextrin on cryosurvival and fertility of cryopreserved carp (Cyprinus carpio) sperm. Cryobiolgy, v.70, p.190-194, 2015. 